\title{
Methods for Calculation of Self-Shielded Cross Sections of Fully Ceramic Micro-Encapsulated Fuel Double Heterogeneous System
}

\author{
Qingming He \\ School of Nuclear Science and Technology, Xi'an Jiaotong University, Xi'an, Shaanxi, China \\ qingming_he@xjtu.edu.cn \\ Wen Yin, Zhouyu Liu, Tiejun Zu, Liangzhi Cao, Hongchun Wu \\ School of Nuclear Science and Technology, Xi'an Jiaotong University, Xi'an, Shaanxi, China
}

\begin{abstract}
Fully ceramic micro-encapsulated (FCM) fuel is a kind of fuel that employs tri-structural isotropic (TRISO) particles to enhance safety. The FCM fuel assembly is a double heterogeneous system. The conventional self-shielding calculation methods cannot treat the DH effect. In this paper, three methods based on equivalent homogenization of the TRISO particle and the matrix are studied and compared: the hyper-fine energy group cross sections (XSs) homogenization based hyper-fine energy group method (HHM), the hyper-fine energy group XSs homogenization based subgroup method (HSM) and the subgroup XSs homogenization based subgroup method (SSM). These methods are implemented in a high-fidelity neutronics code NECP-X. Numerical results show that these methods are able to treat the double heterogeneity of the FCM fuel. The precision of the HHM and HSM is higher than that of the SSM.
\end{abstract}

Key Words: FCM fuel; double heterogeneity; hyper-fine energy group method; subgroup method; NECP-X

\section{INTRODUCTION}

The fully ceramic micro-encapsulated (FCM) fuel is a kind of promising accident-tolerant fuel (ATF) [1]. It is promising to enhance the ability to barrier fission product dispersion, mechanical stability and thermal conductivity. The FCM fuel is composed of tri-structural isotropic (TRISO) particles and matrix. A TRISO particle consists of fuel kernel and several protective layers. The TRISO particles are randomly distributed in the matrix of the FCM fuel. This new design has the so-called double heterogeneity (DH). The first-level heterogeneity is the heterogeneity of the TRISO particles and the surrounding matrix; the second-level heterogeneity is the heterogeneity of the FCM fuel and the moderator.

The DF effect introduces challenges for the neutronics calculation based on the deterministic method. In the deterministic method, conventionally, material is assumed to be piece-wise homogeneous within a mesh. However, this approximation cannot consider TRISO particles. To address the challenge, different methods are proposed.

The first method is the DH transport method proposed by Sanchez [2] and Hebert [3]. This kind of method derives the neutron balance equation based on the statistical treatment. The existence of the TRISO particles are directly considered without homogenization of the material. The second method is the Dancoff method [4-6]. This method employs equivalence theory, subgroup method or hyper-fine energy group method to consider the first-level heterogeneity. Then the Dancoff factor is used to consider the second-level heterogeneity. The third method is the disadvantage factor (DF) method. The DFs are calculated based on 1-D spherical or cylindrical TRISO-matrix model. Then the DFs are used to homogenize the TRISO particles and the matrix. After homogenization, conventional methods like collision probability method and method of characteristic can be used to solve the neutron transport equation. 
The DF method can be easily implemented in neutronics calculation codes with small modifications. In this paper, three methods based on equivalent homogenization of the TRISO particle and the matrix are studied and compared. The first is the hyper-fine energy group cross sections (XSs) homogenization based hyper-fine energy group method (HHM). This method homogenizes the hyper-fine energy group XSs of the TRISO particle and the matrix. Then the hyper-fine energy group neutron slowing-down equation with the homogenized XSs is solved to obtain the self-shielded XSs. The second is the hyperfine energy group XSs homogenization based subgroup method (HSM). The hyper-fine energy group XSs are homogenized as the HHM. The resonance XS table and physical probability table are constructed on-the-fly based on the homogenized hyper-fine energy group XSs. The consistent subgroup fixed-source equations are solved and the self-shielded XSs are obtained. The third is the subgroup XSs homogenization based subgroup method (SSM). This method homogenizes the subgroup XSs of the TRISO particle and the matrix. Then the consistent subgroup fixed-source equations are solved to obtain the self-shielded XSs. These methods are implemented in the self-shielding module of NECP-X [7], which is a high-fidelity deterministic neutronics code, like DeCART, MPACT and nTRACER.

The rest of the paper is organized as follows. The theory of the HHM, HSM and SSM are described in Section 2. These three methods are compared based on the same nuclear data library and code system in Section 3.The conclusions are given in Section 4.

\subsection{Overview of the Methods}

\section{THEORY AND MODEL}

The HHM, HSM and SSM are based on the global-local self-shielding calculation scheme. There are three steps in the global-local self-shielding calculation scheme: the global calculation, the coupling calculation and the local calculation. In the global calculation, the neutron current method is adopted to calculate the Dancoff correction factors of each pin cell. In the coupling calculation, an equivalent 1-D pin cell model is constructed according to preservation of the Dancoff correction factor for each pin cell. The equivalent 1-D pin cell model is shown in Figure 1. In the local calculation, HHM, HSM or SSM are employed to calculate the self-shielding XSs considering DH effect based on the equivalent 1-D pin cell model.
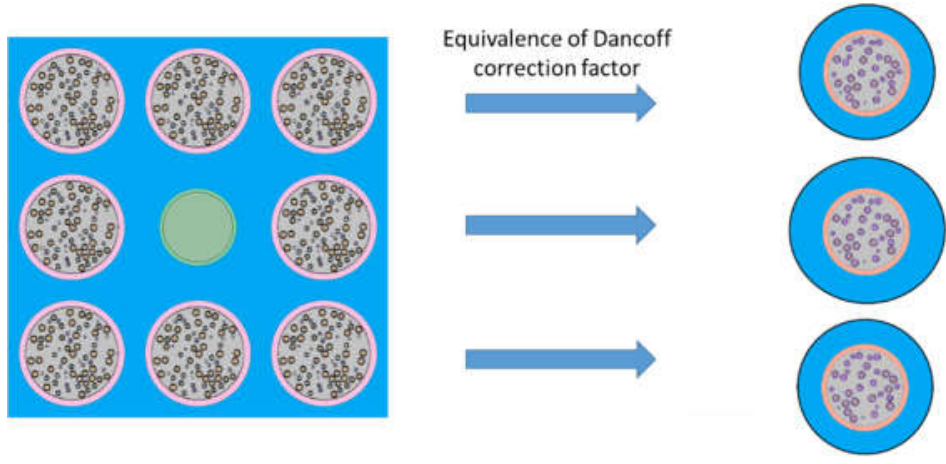

Figure 1 Equivalent 1-D pin cells of the FCM fuel lattice

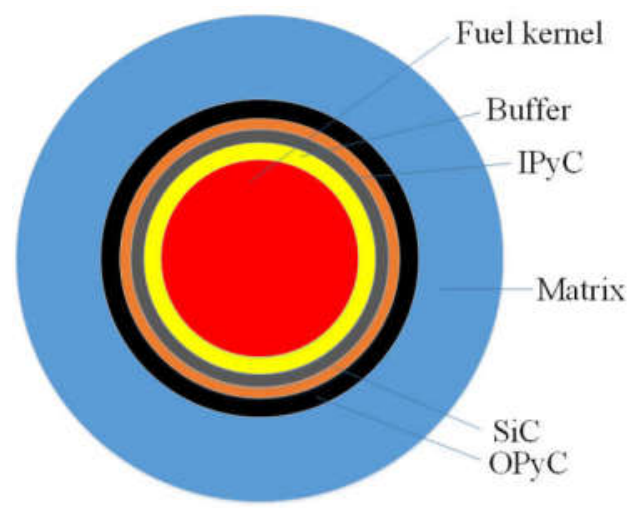

Figure 2 Volume-equivalent 1-D

TRISO-matrix model

\subsection{Hyper-Fine Energy Group Cross Sections Homogenization Based Hyper-Fine Energy Group}

\section{Method}

The hyper-fine energy group DFs are calculated based on the volume-equivalent 1-D TRISO -matrix model shown in Figure 2. The volume of the matrix is calculated according to: 


$$
V_{\text {model,matrix }}=\frac{(1-F) V_{\text {fuel }}}{N_{\text {TRISO }}}
$$

where $F$ is the packing factor; $V_{\text {fuel }}$ is the volume of the fuel region of the pin cell; $N_{\text {TRISO }}$ is the number of TRISO particles. The hyper-fine energy group neutron slowing-down equation is solved based on the volume-equivalent 1-D TRISO-matrix model. According to the preservation of the reaction rate, the homogenized XSs of the TRISO particle and matrix can be written as:

$$
\tilde{\Sigma}_{x, h}=\frac{\sum_{i} V_{i} \phi_{i, h} \Sigma_{x, i, h}}{\sum_{i} V_{i} \bar{\phi}_{h}}=\sum_{i} S_{i, h} f_{i} \Sigma_{x, i, h}
$$

where $x$ is the reaction type; $h$ is the index of the hyper-fine energy group; $i$ is the index of the layer; $\tilde{\Sigma}_{x, h}$ is the homogenized macroscopic XS; $\phi_{i, h}$ is the hyper-fine energy group flux; $\Sigma_{x, i, h}$ is the macroscopic XS of $i$-th layer; $\bar{\phi}_{h}$ is the average flux; $S_{i, h}=\frac{\phi_{i, h}}{\bar{\phi}_{h}}$ is the hyper-fine energy group DF; $f_{i}=\frac{V_{i}}{\sum_{i} V_{i}}$ is the volume fraction. The TRISO particle and the matrix are homogenized in Eq. (2). Therefore, the hyperfine energy group neutron slowing down equation of the equivalent 1-D pin cell model can be solved, the self-shielded XSs can be obtained by reaction rate conservation.

\subsection{Hyper-Fine Energy Group Cross Sections Homogenization Based Subgroup Method}

In the pseudo-resonant-nuclide subgroup method [8-9], the pseudo resonant nuclide is defined by mixing all the resonant nuclides in the fuel region according to the number densities. The resonance XS table is obtained by solving the 0 -D neutron slowing-down equations of the resonant nuclide. If there exist TRISO particles, the hyper-fine energy group DFs $S_{i, h}$ are calculated based on the volume-equivalent 1D TRISO-matrix model. The homogenized microscopic hyper-fine energy group XSs of the resonant nuclides and the pseudo resonant nuclide are obtained by applying the DFs. The 0-D neutron slowingdown equations over a range of background XSs with the homogenized XSs of the resonant nuclides and the pseudo resonant nuclide. The resonance XS table is expressed as:

$$
\tilde{\sigma}_{x, k, g}\left(\sigma_{\mathrm{b}}\right)=\frac{\int_{\Delta u_{g}} \tilde{\sigma}_{x, k}(u) \phi\left(u, \sigma_{\mathrm{b}}\right) \mathrm{d} u}{\int_{\Delta u_{g}} \phi\left(u, \sigma_{\mathrm{b}}\right) \mathrm{d} u} \approx \frac{\sum_{h} \tilde{\sigma}_{x, k, h} \phi_{h}\left(\sigma_{\mathrm{b}}\right)}{\sum_{h} \phi_{h}\left(\sigma_{\mathrm{b}}\right)}
$$

where $\sigma_{\mathrm{b}}$ is the background XS; $u$ is the lethargy; $\widetilde{\sigma}_{x, k}(u)$ is the homogenized microscopic XS. Eq. (3) can be written in subgroup form based on the intermediate resonance approximation as:

$$
\tilde{\sigma}_{x, k, g}\left(\sigma_{b}\right)=\frac{\sum_{s} \frac{\tilde{\sigma}_{x, k, g, s} w_{g, i} \sigma_{\mathrm{b}}}{\widetilde{\sigma}_{\text {pseudo,inter }, g, s}+\sigma_{\mathrm{b}}}}{\sum_{s} \frac{w_{g, i} \sigma_{\mathrm{b}}}{\widetilde{\sigma}_{\text {pseudo,inter }, g, s}+\sigma_{\mathrm{b}}}}
$$

where $s$ is the subgroup index; $\tilde{\sigma}_{x, k, g, s}$ is the subgroup XS; $w_{g, i}$ is the subgroup weight; $\tilde{\sigma}_{\text {pseudo,inter, }, s,}$ is the intermediate subgroup XS of the pseudo resonant nuclide. The subgroup parameters are obtained by 
fitting Eq. (4). Then the subgroup flux is obtained by solving the subgroup neutron slowing-down equation and the self-shielded XS is calculated by:

$$
\widetilde{\sigma}_{x, k, m, g}^{\mathrm{eff}}=\frac{\sum_{s} \tilde{\sigma}_{x, k, g, s} \phi_{m, g, s}}{\sum_{s} \phi_{m, g, s}}
$$

\subsection{Subgroup Cross Sections Homogenization Based Subgroup Method}

In the SSM, the resonance XS table is obtained without considering DH effect:

$$
\sigma_{x, k, g}\left(\sigma_{\mathrm{b}}\right)=\frac{\int_{\Delta u_{g}} \sigma_{x, k}(u) \phi\left(u, \sigma_{\mathrm{b}}\right) \mathrm{d} u}{\int_{\Delta u_{g}} \phi\left(u, \sigma_{\mathrm{b}}\right) \mathrm{d} u} \approx \frac{\sum_{h} \sigma_{x, k, h} \phi_{h}\left(\sigma_{\mathrm{b}}\right)}{\sum_{h} \phi_{h}\left(\sigma_{\mathrm{b}}\right)}
$$

Then the subgroup probability table is obtained by fitting:

$$
\sigma_{x, k, g}\left(\sigma_{b}\right)=\frac{\sum_{s} \frac{\sigma_{x, k, g, s} w_{g, i} \sigma_{\mathrm{b}}}{\sigma_{\mathrm{pseudo}, \text { inter }, g, s}+\sigma_{\mathrm{b}}}}{\sum_{s} \frac{w_{g, i} \sigma_{\mathrm{b}}}{\sigma_{\mathrm{pseudo,inter}, g, s}+\sigma_{\mathrm{b}}}}
$$

The cylindrical TRISO-matrix model is used to calculate the subgroup DF. The subgroup DF is calculated by:

$$
S_{i, g, s}=-\frac{\ln \left(p_{g, s}\right)}{L} \frac{p_{i, g, s}}{1-p_{g, s}} \frac{1}{f_{i}} \frac{1}{\Sigma_{\mathrm{t}, i, g, s}}
$$

where $p_{g, s}$ is the penetrating probability; $L$ is the height of the cylinder model; $p_{i, g, s}$ is the first collision probability in the $i$-th layer; $\Sigma_{\mathrm{t}, i, g, s}$ is the macroscopic total subgroup XS. The homogenized macroscopic subgroup XS is written as:

$$
\tilde{\Sigma}_{x, m, g, s}=\sum_{i} f_{i} \tilde{\Sigma}_{x, i, g, s}=\sum_{i} f_{i} S_{i, g, s} \Sigma_{x, i, g, s}
$$

The subgroup neutron slowing-down equation is formulated with the homogenized macroscopic subgroup XS. The subgroup flux is obtained by solving the subgroup neutron slowing-down equation and the self-shielded XS is calculated according to Eq. (5). It should be noted that the microscopic subgroup $\mathrm{XS}$ is:

$$
\tilde{\sigma}_{x, k, g, s}=S_{i=1, h} \sigma_{x, k, g, s}
$$

\section{NUMERICAL RESULTS}

The HHM, HSM and SSM are implemented based on the high-fidelity neutronics code NECP-X. The results of NECP-X are compared to those of Serpent. The nuclear data library of NECP-X and Serpent are both based on the ENDF-B/VII.0.

\subsection{Cases with Different Packing Factors}

The material and the geometry of the TRISO particles are given in Table I. The material and the geometry of the pin cell problems are given in Table II. The temperature of all materials is $600 \mathrm{~K}$ and the 
fuel enrichment is $15 \%$. The $k_{\text {inf }}$ calculated by different methods are compared to those calculated by Serpent in Table III. The values within parentheses are standard error. The biases are calculated by:

$$
B I A S=\left(k_{\text {inf,cmp }}-k_{\text {inf,ref }}\right) \times 10^{5}
$$

It is found that the biases of the HHM and HSM are smaller than those of the SSM.

Table I Material and geometry of TRISO particle

\begin{tabular}{|c|c|}
\hline Material & Outer radius $(\mathrm{cm})$ \\
\hline Fuel & 0.0250 \\
\hline Buffer & 0.0340 \\
\hline IPyC & 0.0380 \\
\hline SiC & 0.0415 \\
\hline OPyC & 0.0455 \\
\hline
\end{tabular}

Table II Material and geometry of the pin cell problems

\begin{tabular}{|c|c|c|}
\hline Material & Outer radius $(\mathrm{cm})$ & Pin pitch $(\mathrm{cm})$ \\
\hline SiC\&TRISO & 0.6252 & - \\
\hline He & 0.6337 & - \\
\hline Cladding & 0.6907 & 1.6500 \\
\hline Water & - & - \\
\hline
\end{tabular}

Table III Comparison of $\boldsymbol{k}_{\text {inf }}$ for different packing factors

\begin{tabular}{|c|c|c|c|c|c|}
\hline \multirow{2}{*}{ Case } & \multirow{2}{*}{ Packing factor } & \multirow{2}{*}{ Reference $k_{\text {inf }}$} & \multicolumn{3}{|c|}{ Bias (pcm) } \\
\cline { 3 - 5 } & & & HHM & HSM & SSM \\
\hline $1-1$ & 0.05 & $0.86532(0.00011)$ & 45 & 25 & 119 \\
\hline $1-2$ & 0.15 & $1.34138(0.00006)$ & 152 & 138 & 459 \\
\hline $1-3$ & 0.25 & $1.48857(0.00005)$ & 19 & -8 & 458 \\
\hline $1-4$ & 0.35 & $1.55110(0.00004)$ & -49 & -104 & 446 \\
\hline
\end{tabular}

\subsection{Cases with Different Fuel Enrichments}

The material and the geometry of the cases are given in Table I and Table II. The packing factor is 0.35 . The temperature of all materials is $600 \mathrm{~K}$. The $k_{\text {inf }}$ calculated by different methods are compared to those calculated by Serpent in Table IV. The biases of SSM are larger than those of HHM and HSM. 
Table IV Comparison of $\boldsymbol{k}_{\text {inf }}$ for different fuel enrichments

\begin{tabular}{|c|c|c|c|c|c|}
\hline \multirow{2}{*}{ Case } & \multirow{2}{*}{ Packing factor } & \multirow{2}{*}{ Reference $k_{\text {inf }}$} & \multicolumn{3}{|c|}{ Bias (pcm) } \\
\cline { 4 - 6 } & & & HHM & HSM & SSM \\
\hline $2-1$ & 5 & $1.18147(0.00006)$ & -65 & -107 & 330 \\
\hline $2-2$ & 10 & $1.43936(0.00005)$ & -56 & -107 & 414 \\
\hline $2-3$ & 20 & $1.61306(0.00004)$ & -65 & -122 & 440 \\
\hline $2-4$ & 25 & $1.65207(0.00004)$ & -70 & -126 & 446 \\
\hline
\end{tabular}

\subsection{Cases with Different Temperatures}

The material and the geometry of the cases are given in Table I and Table II. The packing factor is 0.35 and the fuel enrichment is $15 \%$. The temperatures of different materials of these cases are given in Table V. The $k_{\text {inf }}$ calculated by different methods are compared to those calculated by Serpent in Table VI. The biases of the SSM are the largest.

Table V Temperature of different materials

\begin{tabular}{|c|c|c|c|c|}
\hline \multirow{2}{*}{ Case } & \multicolumn{4}{|c|}{ temperature (K) } \\
\cline { 2 - 5 } & Fuel & Gap & Cladding & Moderator \\
\hline $3-1$ & 300 & 300 & 300 & 300 \\
\hline $3-2$ & 900 & 600 & 600 & 600 \\
\hline $3-3$ & 1200 & 600 & 600 & 600 \\
\hline
\end{tabular}

Table VI Comparison of $\boldsymbol{k}_{\text {inf }}$ for different temperatures

\begin{tabular}{|c|c|c|c|c|c|}
\hline \multirow{2}{*}{ Case } & \multirow{2}{*}{ Temperature $(\mathrm{K})$} & \multirow{2}{*}{ Reference $k_{\text {inf }}$} & \multicolumn{3}{|c|}{ Bias (pcm) } \\
\cline { 4 - 6 } & & & HHM & HSM & SSM \\
\hline $3-1$ & 300 & $1.55082(0.00004)$ & -70 & 147 & 532 \\
\hline $3-2$ & 900 & $1.54157(0.00005)$ & 194 & -202 & 402 \\
\hline $3-3$ & 1200 & $1.53288(0.00005)$ & -136 & -225 & 417 \\
\hline
\end{tabular}

\subsection{Cases with big TRISO particle}

The outer radius of the TRISO particles is $0.08 \mathrm{~cm}$ for these cases. Other material and geometry parameters of the cases are given in Table I and Table II. The fuel enrichment is $15 \%$ and the temperature of all the material is $600 \mathrm{~K}$. The $k_{\text {inf }}$ calculated by different methods are compared to those calculated by Serpent in Table VII. For case 4-1, the packing factor of which is the smallest, the biases of all methods are large. For other cases, the biases of HHM and HSM are small. 
Table VII Comparison of $\boldsymbol{k}_{\text {inf }}$ for cases with big TRISO particle

\begin{tabular}{|c|c|c|c|c|c|}
\hline \multirow{2}{*}{ Case } & \multirow{2}{*}{ Packing factor } & \multirow{2}{*}{ Reference $k_{\text {inf }}$} & \multicolumn{3}{|c|}{ Bias (pcm) } \\
\cline { 4 - 6 } & & & HHM & HSM & SSM \\
\hline $4-1$ & 0.05 & $0.85363(0.00011)$ & -481 & -527 & -434 \\
\hline $4-2$ & 0.15 & $1.33955(0.00006)$ & 205 & 153 & 507 \\
\hline $4-3$ & 0.25 & $1.49229(0.00004)$ & -15 & -92 & 485 \\
\hline $4-4$ & 0.35 & $1.53088(0.00004)$ & -103 & -197 & 468 \\
\hline
\end{tabular}

\subsection{Cases with the Pin Radius to be $0.5018 \mathrm{~cm}$}

The material and geometry of the TRISO particles are given in Table I. The material and geometry of the pin cell are given in Table VIII. The fuel enrichment is $15 \%$ and the temperature of all materials is 600 $\mathrm{K}$. The $k_{\text {inf }}$ calculated by different methods are compared to those calculated by Serpent in Table IX. The biases of the SSM is the largest.

Table VIII Material and geometry of cases with the pin radius to be $0.5018 \mathrm{~cm}$

\begin{tabular}{|c|c|c|}
\hline Material & Outer radius $(\mathrm{cm})$ & Pin pitch $(\mathrm{cm})$ \\
\hline SiC\&TRISO & 0.5018 & - \\
\hline $\mathrm{He}$ & 0.5103 & - \\
\hline Cladding & 0.5673 & 1.4300 \\
\hline
\end{tabular}

Table IX Comparison of $\boldsymbol{k}_{\text {inf }}$ with different packing factors with the pin radius to be $0.5018 \mathrm{~cm}$

\begin{tabular}{|c|c|c|c|c|c|}
\hline \multirow{2}{*}{ Case } & \multirow{2}{*}{ Packing factor } & \multirow{2}{*}{ Reference $k_{\text {inf }}$} & \multicolumn{3}{|c|}{ Bias (pcm) } \\
\cline { 4 - 6 } & & & HHM & HSM & SSM \\
\hline $5-1$ & 0.05 & $0.77435(0.00013)$ & 18 & 3 & 71 \\
\hline $5-2$ & 0.15 & $1.27231(0.00006)$ & 183 & 167 & 438 \\
\hline $5-3$ & 0.25 & $1.44564(0.00005)$ & 43 & 15 & 434 \\
\hline $5-4$ & 0.35 & $1.52636(0.00004)$ & -45 & -96 & 415 \\
\hline
\end{tabular}

4. CONCLUSIONS

The fully ceramic micro-encapsulated (FCM) fuel has TRISO particles statistically distributed in matrix, which constitute double heterogeneous (DH) effect. The DH effect is a challenge for conventional selfshielding calculation methods. In this paper, three methods based on equivalent homogenization of the 
TRISO particle and the matrix are studied: the hyper-fine energy group cross sections (XSs) homogenization based hyper-fine energy group method (HHM), the hyper-fine energy group XSs homogenization based subgroup method (HSM) and the subgroup XSs homogenization based subgroup method (SSM). These methods are compared for cases with different packing factors, fuel enrichments, temperatures, TRISO particle radius and pin radius. The numerical results show that the precision of the HHM and HSM is higher than that of the SSM.

\section{ACKNOWLEDGMENTS}

This work is supported by the National Natural Science Foundation of China (No. 11805148) and China Postdoctoral Science Foundation (No. 2019M653653 and 2019T120916).

\section{REFERENCES}

1. J. J. Powers, W. J. Lee, F. Venneri, et al. "Fully ceramic microencapsulated (FCM) replacement fuel for LWRs.” Oak Ridge National Laboratory, ORNL/TM-2013/173, KAERI/TR-5136/2013 (2013).

2. R. Sanchez, G. C. Pomraning. "A statistical analysis of the double heterogeneity problem." Annals of Nuclear Energy, 18(7), pp. 371-395 (1991).

3. A. Hebert. A collision probability analysis of the double-heterogeneity problem. Nuclear Science and Engineering, 115, pp. 177-184 (1993).

4. E. E. Bende, A. H. Hogenbirk, J. L. Kloosterman, et al. "Analytical calculation of the average Dancoff factor for a fuel kernel in a pebble bed high-temperature reactor." Nuclear Science and Engineering, 133, pp. 147-162 (1999).

5. H. Kim, S. Choi, M. Park, et al. Extension of double heterogeneity treatment method for coated TRISO fuel particles. Annals of Nuclear Energy, 99, pp. 124-135 (2017).

6. W. Yin, T. Zu, Q. He, et al. "Muti-group effective cross section calculation method for fully ceramic micro-encapsulated fuel." Annals of Nuclear Energy, 125, pp. 26-37 (2019).

7. C. Chen, Z. Liu, Z, Chen, et al. "A new high-fidelity neutronics code NECP-X." Annals of Nuclear Energy, 116, pp. 417-428 (2018).

8. Z. Liu, Q. He, T. Zu, et al. "The pseudo-resonant-nuclide subgroup method based global-local selfshielding calculation scheme." Journal of Nuclear Science and Technology, 55(2), pp. 217-228 (2018).

9. Z. Liu, Q. He, X. Wen, et al. "Improvement and optimization of the pseudo-resonant-nuclide subgroup method in NECP-X." Progress in Nuclear Energy, 103, pp. 60-73 (2018). 